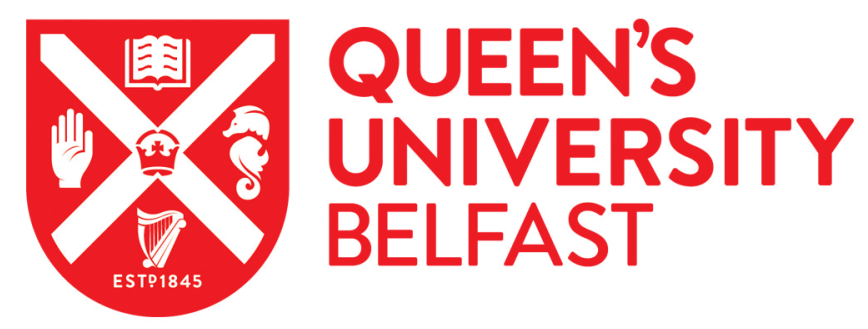

\title{
Real-time Optimised Path Planning and Energy Consumption for Data Collection in UAV-aided Intelligent Wireless Sensing
}

Huynh, D. V., Do-Duy, T., Nguyen, L. D., Le, M. T., Vo, N-S., \& Duong, T. Q. (2021). Real-time Optimised Path Planning and Energy Consumption for Data Collection in UAV-aided Intelligent Wireless Sensing. IEEE Transactions on Industrial Informatics. https://doi.org/10.1109/TII.2021.3114358

Published in:

IEEE Transactions on Industrial Informatics

Document Version:

Peer reviewed version

Queen's University Belfast - Research Portal:

Link to publication record in Queen's University Belfast Research Portal

Publisher rights

(c) 2021 IEEE.

This work is made available online in accordance with the publisher's policies. Please refer to any applicable terms of use of the publisher.

\section{General rights}

Copyright for the publications made accessible via the Queen's University Belfast Research Portal is retained by the author(s) and / or other copyright owners and it is a condition of accessing these publications that users recognise and abide by the legal requirements associated with these rights.

Take down policy

The Research Portal is Queen's institutional repository that provides access to Queen's research output. Every effort has been made to ensure that content in the Research Portal does not infringe any person's rights, or applicable UK laws. If you discover content in the Research Portal that you believe breaches copyright or violates any law, please contact openaccess@qub.ac.uk. 


\title{
Real-time Optimised Path Planning and Energy Consumption for Data Collection in UAV-aided Intelligent Wireless Sensing
}

\author{
Dang Van Huynh, Tan Do-Duy, Long D. Nguyen, Minh-Tuan Le, Nguyen-Son Vo, and Trung Q. Duong
}

\begin{abstract}
In this paper, we consider a new UAV-aided intelligent wireless sensing scheme where the UAVs are deployed for smart sensing and collecting data from Internet-of-Things (IoT) devices. In particular, we propose optimal UAVs' path planing approaches for minimising the completion time and total energy consumption of the UAVs' deployment for data collection. Two optimal schemes, namely optimal energy consumption by peer-topeer UAV-IoT sensing networks and optimal energy consumption by clustering UAV-IoT sensing networks, are considered. The lowcomplexity procedures of our advanced optimisation techniques are suitably applied to disaster relief networks when the solving time must be strictly adhered to. Our real-time optimisation algorithms result in low computational complexity with fast deployment and low processing time for solving the problem of tracking and gathering sensor data, i.e., in very short time (milliseconds). Through simulations results we demonstrate that our proposed approaches in UAV-aided intelligent IoT wireless sensing are suitable for time-critical mission applications such as emergency communications, public safety, and disaster relief networks.
\end{abstract}

Index Terms-Internet of Things (IoT), practical optimization, unmanned ariel vehicles (UAV), real-time optimisation, wireless communication, wireless sensing.

\section{INTRODUCTION}

In the context of the Internet-of-Things (IoT), data collection is one of the most important functionalities. Traditional approaches for data collection have used static sensors and IoT gateways to forward the collected data to a central database such as a server in the cloud. However, in situations where the targets to monitor are placed in remote or difficult-toreach areas or in disaster scenarios, these traditional wireless

Dang Van Huynh and Trung Q. Duong are with the School of Electronics, Electrical Engineering and Computer Science, Queen's University Belfast, Belfast, BT7 1NN, UK (email:\{dhuynh01,trung.q.duong\}@qub.ac.uk).

Tan Do-Duy is with the Department of Computer and Communications Engineering, HCMC University of Technology and Education, 700000 Vietnam (email: tandd@hcmute.edu.vn).

Long D. Nguyen is with the Department of Engineering, Dong Nai University, Bien Hoa, Dong Nai 810000, Vietnam (email:dinhlonghcmut@gmail.com).

Minh-Tuan Le is with MobiFone Research and Development Center, MobiFone Corporation, Hanoi, Vietnam (e-mail:tuan.minh@mobifone.vn).

Nguyen-Son Vo is with the Institute of Fundamental and Applied Sciences, Duy Tan University, Ho Chi Minh City 700000, Vietnam, and also with the Faculty of Electrical-Electronic Engineering, Duy Tan University, Da Nang 550000, Vietnam (e-mail: vonguyenson@ duytan.edu.vn)

Corresponding author is Trung Q. Duong. This work was supported in part by the U.K. Royal Academy of Engineering (RAEng) under the RAEng Research Chair and Senior Research Fellowship scheme Grant RCSRF2021 \11 \41. A part of this paper has been accepted for presentation at the International Wireless Communications and Mobile Computing Conference (IWCMC), Tangier, Morocco; June $24-28,2019$. sensing solutions would exhibit their disadvantages. Recently, unmanned aerial vehicles (UAVs) have been considered as an advanced approach for smart sensing and data collection.

One of UAVs' benefits is their altitude when they operate, which brings about the dominance of line-of-sight (LoS) connections in UAV communication [1]. This makes UAV-aided communications more promising in terms of performance and cost efficiency, thus suitable for the deployment of IoT applications [2], [3]. For instance, UAVs can expand network coverage and improve service quality for more IoT devices by dynamically being deployed in the area. In IoT applications, UAV-aided wireless sensing can deal with the high demands of flexibility, scalability, and reliability required of data gathering techniques. Hence, there are many UAV use cases such as in environmental remediation, agricultural monitoring systems [4], automated inspection [5], or performance of dangerous tasks in disaster relief [6]-[8]. In these instances, UAVs can be deployed to track and communicate with ground IoT devices, including wireless sensor nodes or actuators. They also communicate with the central control system which estimates their paths for intelligent wireless sensing and allocates their resources for the mission. In real-time applications, the UAVs will be able to collect and transmit sensor data back to the central system within strict time deadlines [7], [9].

For data collection applications, there is an increased attention in recent research proposing UAV-based solutions with different objectives such as optimal path planning, scheduling [10]-[14]. In [10], a polynomial-time algorithm based on the concept of graph labelling has been proposed to solve the problem of UAV scheduling for data collection with battery recharging. Another UAV path planning for information collection and transmission method has been discussed in [11]. Specifically, rapid collection and reliable transmission of emerging information have been considered for jointly optimising UAVs' 3-D position and transmit power of the UAVs. In [12], joint UAVs' path planning and travel optimisation for energy efficient data gathering has been also addressed. In fact, UAVs path planning has been optimised for data collection from sensors to ensure efficient collection of all data under constrained energy consumption. In [13], the UAV trajectory planning for data collection in time-constrained devices has been investigated by proposing branch, reduce and bound approach. In [14], another UAVs' path planning optimisation problem has been addressed by deep reinforcement learning in stead of solving a conventional optimisation problem.

Despite their huge potential in many practical applications, 
the limited functions in low-cost UAVs poses a challenge to the growth of the market for UAVs. There are still open research issues in terms of practical implementation to fully realise the UAV's potential. For instance, the fast deployment and efficient operation, which aims to provide and maintain high capability and capacity in UAV communications, are very important yet challenging. Specifically, the number of UAVs to be despatched are dependent on the number of IoT devices assigned to them, network topologies, communication protocols, UAVs' onboard energy capacity, and path planning models. Moreover, UAVs' deployment strategies represent many challenges in nonlinear systems, non-convex optimisation problems, and constrained clustering model in UAV networks [6], [15]-[19]. Among them, K-means clustering offers a low-complexity yet quick cluster selection model. However, it is not straightforward to utilise this method and directly apply it to UAV's scenarios with many constraints. In this work, we propose and compare two different approaches, based on (i) peer-to-peer (P2P) networking and (ii) constrained K-means clustering method (CUN) to allocate IoT devices to UAV collectors and deal with the UAVs' path planning and UAV's energy consumption optimisation problems.

Traditionally, the commercial low-cost UAV devices have limited energy capacity, which restricts their wider applications. Current low-cost UAVs do not have enough onboard energy storage for a long flight, i.e., they can normally operate up to an hour in one charge. For UAV communication, due to the dynamically changing environments, the UAV needs to frequently adapt to re-organised networks. It implies that we need to take into account the fast time-varying environment to design the UAV path planning. This process will cost more energy to prolong the stability of the UAV networks. It is a huge challenge for system designers to develop the optimal UAV's deployment with small completion time and optimal energy consumption. As a result, this research issue has attracted great attention from the industry and academia. Very recently, the UAV-aided disaster communications integrated with wireless sensor networks have been proposed in [1]. In particular, we aimed at minimising the UAVs' total flight time to complete data collection from sensor nodes [1]. It is important to implement real-time path planner for UAVs in the context of mission-critical services. The faster the planner executes, the more time is saved in completing UAVs' missions.

In this paper, we take a step further to extend our work in [1] for UAV-aided intelligent wireless sensing by proposing a real-time optimised UAVs' completion time and energy consumption for data collection. More specifically, we deploy a UAV network comprising of multiple UAVs which follow optimised paths for for intelligent wireless sensing and gathering monitored data in mission-critical applications such as disaster relief networks. To tackle the time-critical issue, we propose optimisation algorithms of low complexity that significantly reduce the execution time. Here, we exploit real-time optimisation [8], [20] to support the UAV communications when fast optimal deployment is vitally important. The main contributions of this paper can be summarised as follows:

- We introduce a novel framework for optimising UAVs' path planning in order to minimise the completion time and total energy consumption of UAVs' deployment procedure in data collection missions. This optimisation model takes into account the constraints of real-world UAVs' deployment.

- We propose two approaches to solving the problem, namely, convex optimisation algorithms of lowcomplexity, and the K-means clustering strategy. These approaches find the optimal completion time and total energy consumption under various practical constraints such as the UAVs'coverage region, flying time, and QoS requirements.

- Finally, the numerical results of a wide range of simulations are also provided to prove the reliability of the proposed optimisation framework. These simulation results are helpful for the deployment of UAV-aided intelligent wireless sensing in the future.

The remainder of the paper is organised as follows. In section II, we present the system model and problem formulation. The first approach to solving the problem, namely Peer-toPeer UAV-IoT networks (P2P), is described in Section III. Next, we introduce the K-means clustering scheme called clustering of UAV-IoT devices (CUN) to find the optimal values of total completion time and energy consumption of the UAVs' deployment in Section IV. In Section V, we provide the simulation results to illustrate the impact of the proposed schemes. Finally, conclusions are given in Section VI.

\section{UAV-IOT SYSTEM MODEL AND PROBLEM FORMULATION}

\section{A. System model}

In this paper, we propose to use UAVs to track and collect information from IoT devices. In particular, $M$ UAVs are available for the mission, their tasks are to gather data from $K \gg 1$ static ground IoT devices, $K$ being denoted by the set of $\mathcal{K}=\{1, \ldots, K\}$. As illustrated in Fig. 1, a ground station (control centre) will optimise the path planning for the $M$ UAVs, $\mathcal{M}=\{1, \ldots, M\}$, and control their deployment accordingly. The IoT devices are positioned within a rectangle target area, $\mathcal{A}(\ell, d)$, where $\ell$ and $d$ are respectively its length and width. The location of an IoT device $k, \boldsymbol{q}_{k}=\left[x_{k}, y_{k}, 0\right]^{T}, k \in \mathcal{K}$, is determined by using the Global Positioning System (GPS). All the UAVs and IoT devices are single-antenna equipped.

Let us assume that the UAVs operate over a duration of $T>$ 0 in second (s). At a time instant $t, 0 \leq t \leq T$, the 3D location of a UAV $m$ is denoted as $\boldsymbol{q}_{m}(t)=\left[x_{m}(t), y_{m}(t), h_{m}(t)\right]^{T} \in$ $\mathbb{R}^{3}, m \in \mathcal{M}$. Without loss of generality, we further assume that the time length $T$ can be divided into $N$ equal timeslots denoted by the set $\mathcal{N}=\{1, \ldots, N\}$. Each slot has the elemental time length as $\eta=T / N$, which is sufficiently small, during which the location of the UAV is stable [21]. Consequently, the UAV's trajectory can be expressed as $\boldsymbol{q}_{m}[n]=\left[x_{m}[n], y_{m}[n], h_{m}[n]\right]^{T}, n \in \mathcal{N}$.

As data collectors, the UAVs should start from their parking dock located at $\boldsymbol{q}_{G, m}=\left[x_{G, m}, y_{G, m}, h_{G, m}\right]^{T}$, i.e., $\boldsymbol{q}_{m}[1]=$ $\boldsymbol{q}_{G, m}$, follow their planned paths, collect data from the IoT devices on their way and finish their mission at their parking 
dock $\boldsymbol{q}_{m}\left[N_{m}\right]=\boldsymbol{q}_{G, m}$ where $N_{m}$ is the last time-slot of the operation of a UAV $m$. The trajectory of a UAV is assumed to be within the considered area $\mathcal{A}$ while it tracks the IoT devices and gathers sensor data. The data packets collected from the IoT devices will be transferred to the central station at the end of the UAV's trajectory.

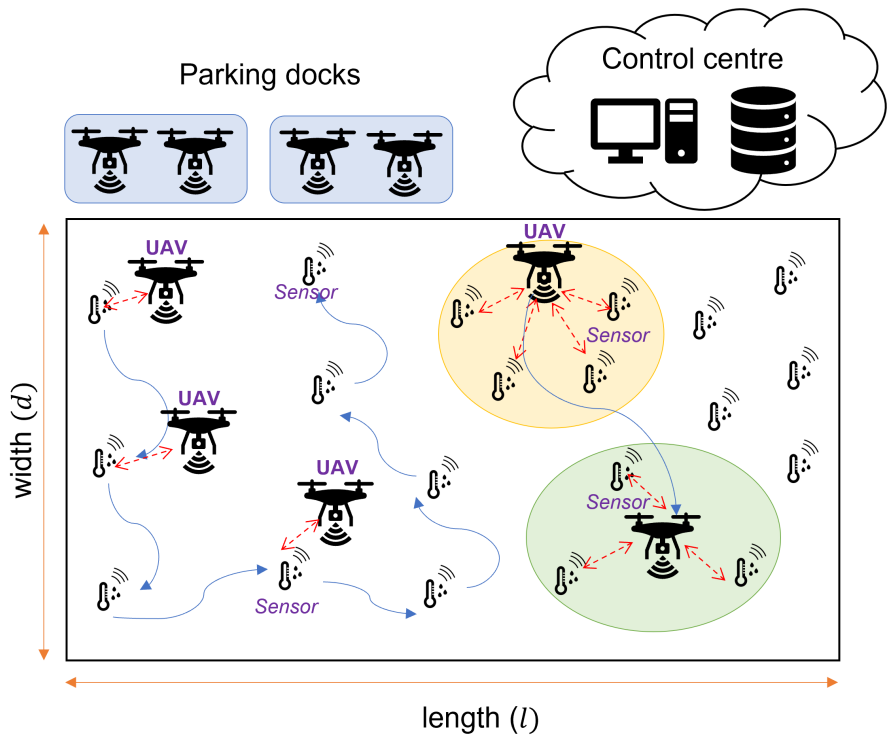

Fig. 1: System model of the UAVs' path planning for data collection in two approaches: Peer-to-peer networking and Kmeans clustering

\section{B. Channel model}

We consider that the air-to-ground wireless channels between the airborne UAVs and ground IoT devices are dominated by LoS links [22]. At the $n$th time-slot of the $m$ th UAV, $R_{m k}[n]$ and $g_{m k}[n]$ are respectively the distance from the $m$ th UAV to the $k$ th IoT device, and the channel power gain of their link, as provided in [1]. The small-scale fading channels can be neglected because of the LoS-dominated UAV communications. Let $\delta_{m, n}$ be the $m$ th UAV's flying time between two consecutive time-slots, and $V$ denote its average speed, then we have:

$$
\delta_{m, n}(\boldsymbol{q})=\frac{\left\|\boldsymbol{q}_{m}[n+1]-\boldsymbol{q}_{m}[n]\right\|}{V}, n=1, \ldots, N .
$$

We define a circular disc with radius $D_{\text {cov }}$ as the coverage region in which the quality-of-service $(\mathrm{QoS})$ requirement is met as a result of the domination of LoS links. The relation between the radius $D_{m, c o v}$ and the altitude of the UAV $m$ can be expressed as

$$
h_{m}[n]=D_{m, \operatorname{cov}} \tan (\theta), \forall n, m,
$$

in which the elevation angle $\theta$ is set at $20.34^{\circ}$ for suburban environment [23]. A UAV can only connect to and gather data from the IoT devices within its coverage range, i.e., the Euclidean distance between the UAV and IoT device must be smaller than the coverage radius $D_{m, \text { cov }}$. This constraint is represented as

$$
D_{m k}[n] \leq D_{m, c o v}, \forall n
$$

For a constant transmit power $\left(P_{k}\right)$ at the $k$ th IoT device, the uplink data rate at the $m$ th $\mathrm{UAV}$ is given as

$$
C_{m k}[n]=B \log _{2}\left(1+g_{m k}[n] P_{k} / \sigma_{m}^{2}\right),
$$

where $B$ is the bandwidth and $\sigma_{m}^{2}$ is the Gaussian noise power at the UAV $m$.

\section{Data collection model}

We assume that the IoT devices forward their data packets, with the size of $\bar{D}_{k}$ by using a standard data rate $\bar{C}_{k}$ to the UAVs. A UAV and IoT devices are connected via an IoT network protocol, i.e., Bluetooth low energy (BLE) or Low power wide area networks (LPWANs) [24]. From (3) and (4), we can rewrite the QoS requirement as

$$
C_{m k}[n] \geq \bar{C}_{k}, \forall n, k, m .
$$

By using eq. (2) in [1], after some manipulations, from (5) we have

$$
h_{m}[n] \leq \sqrt{\frac{\beta_{0} P_{k}}{\sigma_{m}^{2}\left(2^{\bar{C}_{k} / B}-1\right)\left(\tan ^{-2}(\theta)+1\right)}}, \forall n, k, m .
$$

In order to analyse the UAVs' mission completion time, we consider that it is comprised of three types of execution time. Firstly, the time needed for completing data collection from the $K$ IoT devices consists of the connecting time $\left(L_{c o n}\right)$ and the data transferring time $\left(L_{\text {data }}\right)$. Secondly, $\left(L_{\text {tran }}\right)$ is the time needed for the $M$ UAVs to cover their planned path over the $K$ IoT devices - this does not include connecting and transferring time. These time series are defined as

$$
\begin{aligned}
L_{\text {con }} & =\sum_{k \in \mathcal{K}} L_{k, c o n}, \\
L_{\text {data }} & =\sum_{k \in \mathcal{K}} \frac{\bar{D}_{k}}{\bar{C}_{k}}, \\
L_{\text {tran }}(\boldsymbol{q}) & =\sum_{m \in \mathcal{M}} \sum_{n=1}^{N} \delta_{m, n}(\boldsymbol{q}),
\end{aligned}
$$

where $L_{k, \text { con }}$ representing the connecting time between a UAV and the $k$ th IoT device, is randomly distributed in a given time interval; and $\boldsymbol{q}=\left\{\boldsymbol{q}_{1}, \ldots, \boldsymbol{q}_{M}\right\}$.

Thus, the total completion time of the $M$ UAVs in the mission is expressed as

$$
L_{\text {tot }}=L_{\text {con }}+L_{\text {data }}+L_{\text {tran }}(\boldsymbol{q}) .
$$

From that, the minimum number of time-slots the UAVs need to finish collecting the sensor data from the $K$ IoT devices can be calculated as

$$
\bar{N}_{\text {min }}=\left\lceil\frac{L_{\text {con }}+L_{\text {data }}+L_{\text {tran }}}{\eta}\right\rceil,
$$

where $\lceil$.$\rceil is a calculation for rounding a decimal value to an$ approximate integer.

Consequently, the number of UAVs needed to cover all the IoT devices can be obtained as

$$
M \geq \bar{M}_{\min }=\left\lceil\frac{\bar{N}_{\min }}{N}\right\rceil \text {. }
$$


To reflect the limitation of the UAVs' operating time in practice, the total allowable completion time in this model must satisfy the following inequality:

$$
L_{\text {tot }} \leq M T_{\max },
$$

where $T_{\max }$ is the maximum operating time of a UAV. For large-scale scenarios, the value of $L_{t o t}$ can be significantly higher than the period time $T_{\max }$.

To support the overall task completion time of multiple UAVs, the UAV-IoT devices clustering will be investigated in this work. The main benefits of proposing UAV-IoT devices clustering are to reduce the number of UAVs to be used for collecting sensor data and the completion time over a turn of sensor exchange data under a parallel collection scheme by $M$ UAVs. To this end, we consider $M$ UAVs for use as $M$ clusters to cover $K$ IoT devices. Thus, the cluster $m$ (UAV $m$ ) can serve a finite number $K_{m}$ of IoT devices, $\mathcal{K}_{m}=\left\{1, \ldots, K_{m}\right\}$. The values of $K_{m}$ are different amongst the $M$ clusters and depend on the clustering approaches.

Equation (8) can be rewritten to show the completion time of a UAV $m$ that is used in the mission, as follows:

$$
L_{m, \text { tot }}=L_{m, \text { con }}\left(\mathcal{K}_{m}\right)+L_{m, \text { data }}\left(\mathcal{K}_{m}\right)+L_{m, \text { tran }}\left(\boldsymbol{q}_{m}\right),
$$

where

$$
\begin{gathered}
L_{m, \text { con }}\left(\mathcal{K}_{m}\right)=\sum_{k \in \mathcal{K}_{m}} L_{k, \text { con }}, \quad L_{m, \text { data }}\left(\mathcal{K}_{m}\right)=\sum_{k \in \mathcal{K}_{m}} \frac{\bar{D}_{k}}{\bar{C}_{k}}, \\
L_{m, \operatorname{tran}}\left(\boldsymbol{q}_{m}\right)=\sum_{n=1}^{N_{m}} \delta_{m, n}(\boldsymbol{q}) .
\end{gathered}
$$

The completion time constraint of the $m$ th UAV is also expressed as

$$
L_{m, t o t} \leq T_{\max } .
$$

On the other hand, to overcome the strict limitation of energy consumption in UAV communications, an energy model with respect to the required time frame, and an optimisation problem for minimising the total energy consumption of the UAVs will be proposed in the next subsections.

\section{Energy model}

Given a constant UAV's operating power, the energy consumed by the $m$ th UAV can be estimated as

$$
\begin{aligned}
E_{m}\left(\boldsymbol{q}, \mathcal{K}_{m}\right) & =P_{m, \text { ope }} L_{m, \text { tot }} \\
& +P_{m, \text { com }}\left(L_{m, \text { con }}\left(\mathcal{K}_{m}\right)+L_{m, \text { data }}\left(\mathcal{K}_{m}\right)\right)
\end{aligned}
$$

where $P_{m, o p e}$ is the UAV's operating power, and $P_{m, c o m}$ is the energy for UAV communication including establishing connections with $\mathcal{K}_{m}$ IoT devices and data transmission activities. This model is used to represent the energy consumed by the UAV to fly through its planned path and to complete data collection from the $\mathcal{K}_{m}$ IoT devices that are allocated to it.
Based on the time frames as specified in (8) and (12), the total energy consumption $\left(E_{t o t}\left(\boldsymbol{q}_{m}\right)\right)$ (in Joules) of all the $\mathrm{UAVs}$ is given as

$$
E_{t o t}(\boldsymbol{q}, \mathcal{K}, M)=\sum_{m \in M} E_{m}\left(\boldsymbol{q}, \mathcal{K}_{m}\right),
$$

where $\mathcal{K}=\left\{\mathcal{K}_{1}, \ldots, \mathcal{K}_{M}\right\}$. According to a comprehensive energy model for UAV kits, the energy consumption of flying operation at the UAVs and communication protocols for IoT network will be separated by using different batteries. In this work, the power of UAV communications in the IoT network is assumed that it is higher than the power of UAV flying operation. Therefore, it is significant to minimise the total operating power of UAVs in the data collection mission. In this paper, we minimise the total energy consumption of UAV operation by optimising the total operating time of all UAVs.

\section{E. Problem formulation}

Our objective is to minimise the total energy consumption as presented in the equation (15), under the constraints on flying time, coverage region, QoS requirement, and number of UAVs. The problem formulation is described as

$$
\begin{aligned}
\min _{\boldsymbol{q}, \mathcal{K}, M} & E_{\text {tot }}(\boldsymbol{q}, \mathcal{K}, M) \\
\text { s.t. } & (3),(6),(10),(11),(13), \\
& \mathbf{q}_{\min } \leq \mathbf{q}_{m} \leq \mathbf{q}_{\max }, \forall m=1, \ldots, M .
\end{aligned}
$$

Equation (15) shows that the $E_{t o t}$ increases as the $L_{t o t}$ increases and as mentioned above, the total energy consumption of UAVs in a particular mission significant relies on the total flying time. Therefore, in this paper, we minimise the energy consumption of all the UAVs by minimising the total completion time. We assume that the central control system is used to estimate the entire paths of UAVs and all optimisation procedures are executed centrally in the control system. We do not rely on a distributed control scheme because it is more complicated to handle optimisation problem in distributed scheme due to other issues such as data exchanges, data synchronisation, scheduling, etc.,. Additionally, when we implement the distributed control scheme on UAVs that means the UAVs have to take additional computing tasks to optimise their paths, which obviously increases the total energy consumption as well as total completion time of UAVs. The following sections present two proposed approaches to solve (16), namely, optimal energy consumption by peer-to-peer UAV-IoT networks (P2P), and optimal energy consumption by clustering UAV-IoT networks (CUN).

\section{OPTIMAL ENERGY CONSUMPTION BY PEER-TO-PEER UAV-IOT NETWORKS (P2P)}

In this approach, we separate the problem (16) into two subproblems. Firstly, the number of UAVs needed is estimated following the peer-to-peer (P2P) networking scheme between UAVs and IoT devices. Then, a simple trajectory design is implemented to minimise the total energy consumption.

Let us assume that the altitudes of all the UAVs are a constant, i.e., $h_{m}=\bar{H}, \forall m$. We consider the total number 
of time-slots of the $M$ UAVs as $K+2 M$ to reflect $K$ timeslots to collect data from the $K$ IoT devices, and an initial and a final time-slot for each UAV. Further to this, let us define the $m$ th UAV's location at its initial and final time-slot as $\boldsymbol{q}_{m}[0]=\boldsymbol{q}_{m}\left[K_{m}+1\right]=\boldsymbol{q}_{G, m}$.

\section{A. Estimating the number of UAVs}

From (1), the UAVs' total flight time in the P2P path planning over $K$ time-slots can be rewritten as

$$
\delta_{p 2 p}=\sum_{k=1}^{K-1} \delta_{m, k}^{p 2 p}=\sum_{k=1}^{K-1} \frac{\left\|\boldsymbol{q}_{m}[k+1]-\boldsymbol{q}_{m}[k]\right\|}{V},
$$

where $\boldsymbol{q}_{m}[k]=\boldsymbol{q}_{k}$.

We propose to use Algorithm 1 for estimating the UAV number in this P2P approach of UAV-IoT device networking. In the algorithm, $L_{m}^{p 2 p}$ is the completion time of the $m$ th UAV,

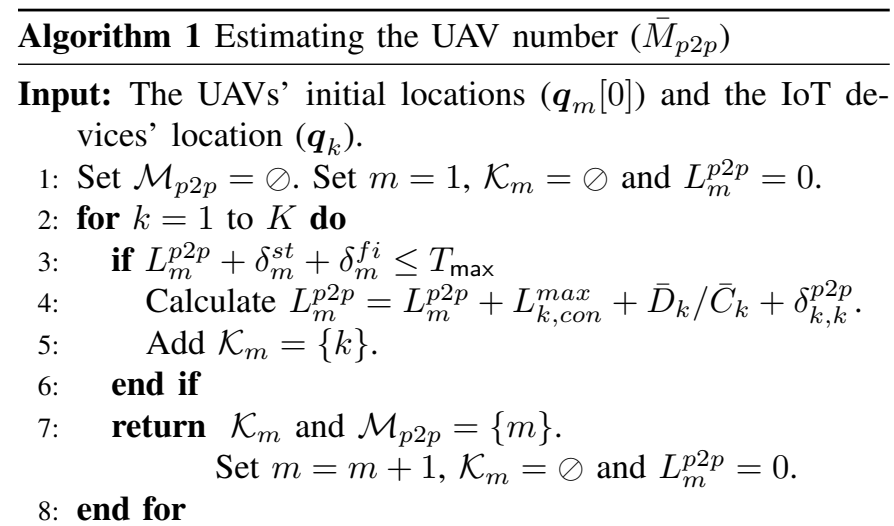

Output: $\left\{\mathcal{M}_{p 2 p}\right\}$ and $\left\{\mathcal{K}_{m}=\left\{1, \ldots, K_{m}\right\}\right\}, \forall m$.

while its initial $\left(\delta_{m}^{s t}\right)$ and final flying time $\left(\delta_{m}^{f i}\right)$ are respectively written as

$$
\begin{aligned}
\delta_{m}^{s t} & =\frac{\left\|\boldsymbol{q}_{m}[0]-\left.\boldsymbol{q}_{k}\right|_{k=1}\right\|}{V}, \\
\delta_{m}^{f i} & =\frac{\left\|\boldsymbol{q}_{m}\left[K_{m}+1\right]-\left.\boldsymbol{q}_{k}\right|_{k=K_{m}}\right\|}{V} .
\end{aligned}
$$

Hence, the $m$ th UAV's completion time $\left(L_{t o t}^{p 2 p}\right)$ is given as

$$
L_{t o t}^{p 2 p}=\sum_{m \in \mathcal{M}_{p 2 p}} L_{m}^{p 2 p},
$$

where

$$
L_{m}^{p 2 p}=\delta_{m}^{s t}+\delta_{m}^{f i}+\sum_{k \in \mathcal{K}_{m}}\left(L_{k, c o n}^{\max }+\frac{\bar{D}_{k}}{\bar{C}_{k}}+\delta_{m, k}^{p 2 p}\right),
$$

such that

$$
L_{m}^{p 2 p} \leq T_{\max }
$$

Thus, the total energy consumption corresponding to the total completion time now can be expressed as:

$$
E_{\text {tot }}^{p 2 p}\left(\boldsymbol{q}, \mathcal{K}, \mathcal{M}_{p 2 p}\right)=\sum_{m \in \mathcal{M}_{p 2 p}} E_{m}^{p 2 p}\left(\boldsymbol{q}, \mathcal{K}_{m}\right),
$$

where

$$
\begin{aligned}
E_{m}^{p 2 p}\left(\boldsymbol{q}, \mathcal{K}_{m}\right) & =\sum_{m \in \mathcal{M}_{p 2 p}} P_{m, o p e} L_{m}^{p 2 p} \\
& +\sum_{m \in \mathcal{M}_{p 2 p}} P_{m, c o m} \sum_{k \in \mathcal{K}_{m}}\left(L_{k, c o n}+\frac{\bar{D}_{k}}{\bar{C}_{k}}\right) .
\end{aligned}
$$

\section{B. Proposed optimisation problem and solving approach}

The subproblem of minimising the total energy consumption (16) without the constraints of the UAV number is given as

$$
\begin{gathered}
\min _{\mathbf{M}, \boldsymbol{q}_{m}} E_{t o t}^{p 2 p}\left(\boldsymbol{q}, \mathcal{K}, \mathcal{M}_{p 2 p}\right) \\
\text { s.t. } \\
(3),(6),(16 c),(22), \\
L_{\text {tot }}^{p 2 p} \leq \bar{M}_{p 2 p} T_{\max } .
\end{gathered}
$$

Unlike the original nonconvex problem (16), the subproblem (24) is a convex problem of low-complexity, whose objective function and constraints are convex. As a result, this subproblem can be handled by using optimisation solvers, such as CVX [25].

\section{OPTIMAL ENERGY CONSUMPTION BY CLUSTERING UAV-IOT NETWORKS (CUN)}

In this approach, we separate the problem (16) into two subproblems. Firstly, the IoT clustering method with QoS constraint (5) will be proposed for partitioning the IoT devices into clusters by constrained K-means clustering procedure. Then, a fast trajectory design is implemented to calculate the number of UAVs needed and minimise the completion time of this scheme.

\section{A. Constrained K-means clustering model}

By following the coverage communication between $\bar{M}_{\text {clus }}$ UAVs and $K$ IoTs devices are divided into multiple groups $\mathcal{K}_{j}=\left\{1, \ldots, K_{j}\right\}, K_{j} \geq 1, j=1, \ldots, J, \bar{M}_{\text {clus }} \leq J$ such that $\sum_{j=1}^{J} K_{j}=K$ and $\mathcal{K}=\left\{\mathcal{K}_{1}, \ldots, \mathcal{K}_{J}\right\}$. Thus, one or more group will transfer their data to the UAV that satisfies the constraints (5) and (11).

We propose an efficient procedure based on constrained Kmeans clustering to group the IoT devices, as in Algorithm 2.

From (1), the new expression for the flying time of a UAV between two adjacent clusters can be written as follows:

$$
\delta_{\text {clus }}=\sum_{j=1}^{J-1} \delta_{m, j}^{\text {clus }}=\sum_{j=1}^{J-1} \frac{\left\|\boldsymbol{q}_{j}[j+1]-\boldsymbol{q}_{j}[j]\right\|}{V},
$$

where $\boldsymbol{q}_{j}[j]=\boldsymbol{\theta}_{j}$ is the location of the $j$ th cluster's centroid. Meanwhile, the initial and final flying time of the UAV over one cluster are respectively expressed as

$$
\begin{aligned}
\delta_{j}^{s t} & =\frac{\left\|\boldsymbol{q}_{G, m}-\left.\boldsymbol{q}_{k}\right|_{k=1, k \in \mathcal{K}_{j}}\right\|}{V}, \\
\delta_{j}^{f i} & =\frac{\left\|\boldsymbol{q}_{G, m}-\left.\boldsymbol{q}_{k}\right|_{k=K_{j}}\right\|}{V} .
\end{aligned}
$$

In addition, the maximum operating time of the UAV has an impact on the number of IoT devices it can serve in each 


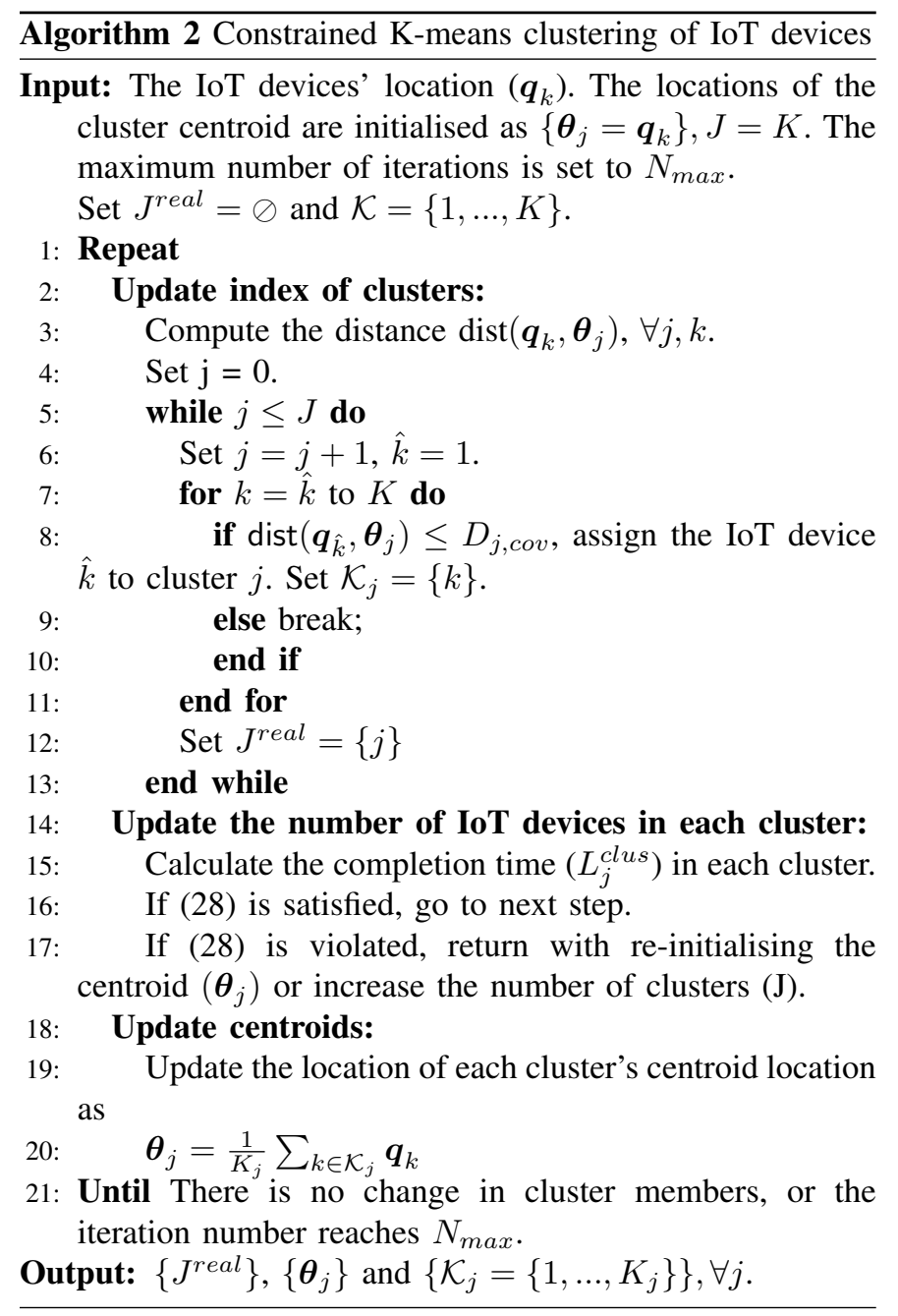

cluster. Thus, to control the number of IoT devices in each cluster, the total UAVs' flying time over their assigned IoT devices must satisfy the following inequality

$$
L_{j}^{\text {clus }}+\delta_{j}^{s t}+\delta_{j}^{f i} \leq T_{\max }
$$

where

$$
L_{j}^{c l u s}=\sum_{k \in \mathcal{K}_{j}}\left(L_{k, c o n}^{\max }+\frac{\bar{D}_{k}}{\bar{C}_{k}}\right)
$$

\section{B. Proposed solving approach}

Let us assume that the location of the UAVs is unchanged while they communicate and collect sensor data from the clusters of IoT devices. We further define a binary variable $\mathbf{a}_{m}=\left[a_{m, j}\right]_{j=1}^{J}$ as

$$
a_{m, j}=\left\{\begin{array}{lc}
1, & \text { UAV serves group } j \\
0, & \text { otherwise. }
\end{array}\right.
$$

In Algorithm 3, we propose a simple method to identify the UAV number and estimate the completion time following the assignment procedure as in (29).

The total flight time for the UAVs to complete data collection is expressed as

$$
L_{\text {tot }}^{\text {clus }}=\sum_{m \in \mathcal{M}_{\text {clus }}} L_{m}^{\text {clus }}
$$

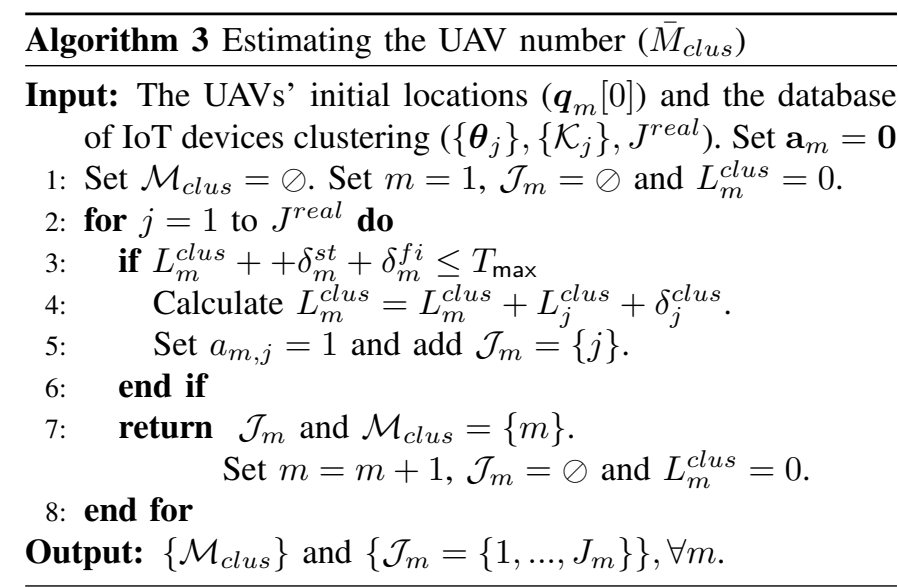

where

$$
L_{m}^{\text {clus }}=\delta_{m}^{s t}+\delta_{m}^{f i}+\sum_{j \in \mathcal{J}_{m}} \delta_{m, j}^{c l u s}+\sum_{k \in \mathcal{K}_{j}}\left(L_{k, c o n}+\frac{\bar{D}_{k}}{\bar{C}_{k}}\right),
$$

$\delta_{m}^{s t}=\frac{\left\|\boldsymbol{q}_{G, m}-\left.\boldsymbol{q}_{j}\right|_{j=1, j \in \mathcal{J}_{m}}\right\|}{V}$ and $\delta_{m}^{f i}=\frac{\left\|\boldsymbol{q}_{G, m}-\left.\boldsymbol{q}_{j}\right|_{j=J_{m}}\right\|}{V}$ such that

$$
L_{m}^{\text {clus }} \leq T_{\max }
$$

Therefore, the total energy consumption from (15) in this approach can be expressed as:

$$
\begin{aligned}
E_{\text {tot }}^{\text {clus }}\left(\boldsymbol{q}, \mathcal{M}_{\text {clus }}\right) & =\sum_{m \in \mathcal{M}_{\text {clus }}} E_{\text {oper }}+\sum_{m \in M} E_{\text {comm }} \\
& =\sum_{m \in \mathcal{M}_{\text {clus }}} P_{m, \text { ope }} \cdot \sum_{m \in \mathcal{M}_{\text {clus }}} L_{m}^{\text {clus }} \\
& +\sum_{m \in \mathcal{M}_{\text {clus }}} P_{m, \text { com }} \cdot \sum_{k \in \mathcal{K}_{j}}\left(L_{k, \text { con }}+\frac{\bar{D}_{k}}{\bar{C}_{k}}\right) .
\end{aligned}
$$

Unlike the P2P networking, this approach results in a noncomplex optimisation problem that can be solved fast and efficiently by using Algorithm 2-3.

\section{Simulation Results}

\section{A. Simulation setup}

To evaluate the performance of our proposed approaches, we used a Windows-based Laptop, $10^{\text {th }}$ Generation Intel® Core $^{\mathrm{TM}}$ i7-10610U with 16GB memory, as the computational platform. The simulations are run on Matlab software, which is a well-known simulator and wildly used in engineering.

For simulations parameters, without loss of generality, we followed the set-up as in [1]. In particular, the connection between the UAVs and IoT devices followed the BLE protocol at the $2.4 \mathrm{GHz}$ frequency, bandwidth $B=1 \mathrm{MHz}$, standard data rate $\left(\bar{C}_{k}=200 \mathrm{kbps}\right)$, and connectivity range of maximum $100 \mathrm{~m}$. The IoT's data packet and constant transmit power were assumed at $\bar{D}_{k}=1 \mathrm{Mbits}$ and $100 \mathrm{~mW}$, respectively. For the network topology, we considered a rectangle network area of $\mathcal{A}(\ell, d)=(2000,200)$. The control station and parking docks 
were assumed to be located at $(100,1000,0)$. The number of IoT devices was set to $K=\{100,200,500,800,1000\}$ for performance evaluation. The average flight speed and maximum operating time of a UAV were $V=10 \mathrm{~m} / \mathrm{s}$ and $T_{\max }=20$ minutes, respectively. The UAVs' altitude was fixed at $H_{m}=[20,100] \mathrm{m}$. The noise power was $-90 \mathrm{~dB}$. Each IoT device's connection time $\left(L_{k, c o n}\right)$ was randomly distributed in the interval $[0.5,5] \mathrm{s}$. The power consumption of a UAV for flying and communication were respectively set to $P_{m}^{o p e r}=300 \mathrm{~W}$ and $P_{m}^{c o m m}=5 \mathrm{~W}$.

\section{B. Simulation results}

1) Completion time minimisation: Fig. 2 plots the minimised completion time in the two proposed approaches against the coverage region $\left(D_{c o v}\right)$ in four different scenarios of the number of IoT devices. In general, the P2P path planning scheme provides lower completion times than the CUN approach. While both schemes provide similar completion time at a small $D_{\text {cov }}$, P2P scheme outperforms its counterpart by a large margin as the coverage region expands. However, at the same number of IoT devices, this margin tends to decrease with increasing the coverage region.
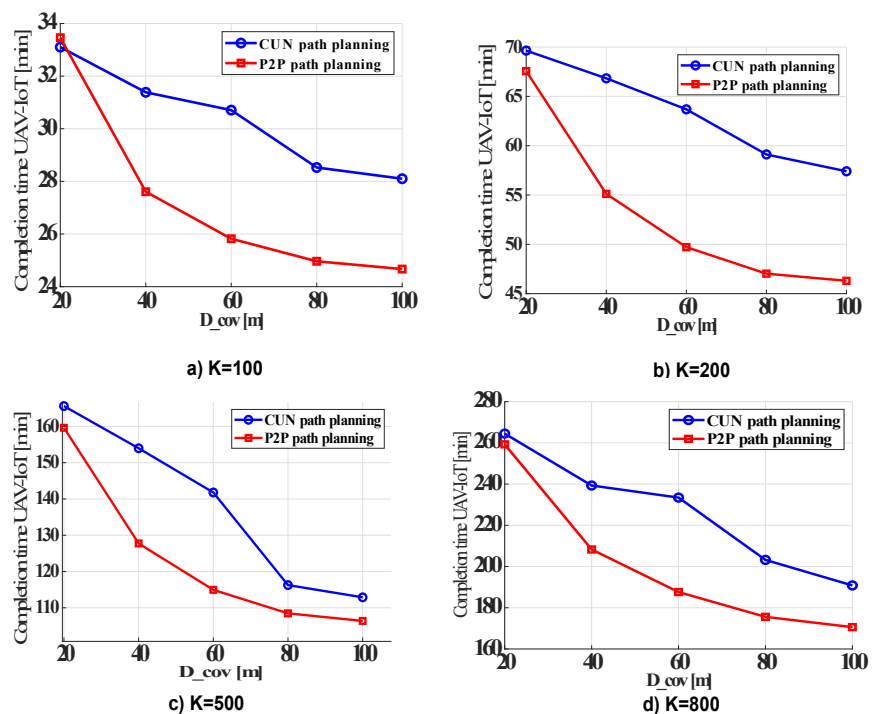

Fig. 2: The completion time versus the coverage region $D_{\text {cov }}$ in P2P and CUN schemes.

2) Total energy consumption minimisation versus coverage region $\left(D_{\text {cov }}\right)$ : In terms of total energy consumption, it looks similar to the total completion time because the completion time plays a key role in UAVs' energy consumption. The results of energy consumption comparisons are presented as in Fig. 3.

The figures illustrate that the $\mathrm{P} 2 \mathrm{P}$ with the optimisation approach saves more energy than the CUN method in most cases, especially in small coverage region (radius from $20 \mathrm{~m}$ to $60 \mathrm{~m}$ ). As can be observed from Fig. 3, when the coverage region of UAVs increases, they spend less time for data collection mission so that their total energy consumption gradually decreases. Interestingly, the difference between two approaches are smaller at the smallest coverage region $(20 \mathrm{~m})$ and the largest coverage region compared to other coverage region values.
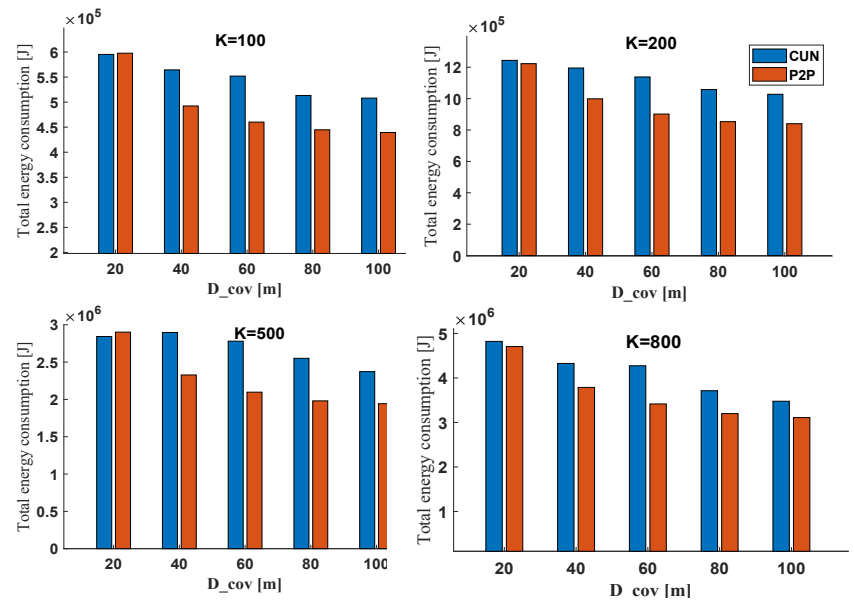

Fig. 3: The total energy consumption versus the coverage region $D_{c o v}$ in P2P and CUN schemes.

3) Total energy consumption versus density levels sensor distribution (K): Fig. 4 was generated to analyse the performance of each approach in UAV planning among various $D_{\text {cov }}$ range at different density levels of sensor distribution.

The figures show that the total energy consumption in both approaches increases when the number of IoT devices increases. Additionally, as the coverage region becomes larger, the UAVs' energy consumption pattern gradually shifts towards supporting data collection. The results also show that the P2P solution is more effective at the large number of IoT devices because its total energy consumption gradually decreases when the coverage region becomes wider, while CUN experiences some fluctuations (e.g., the last green bar).
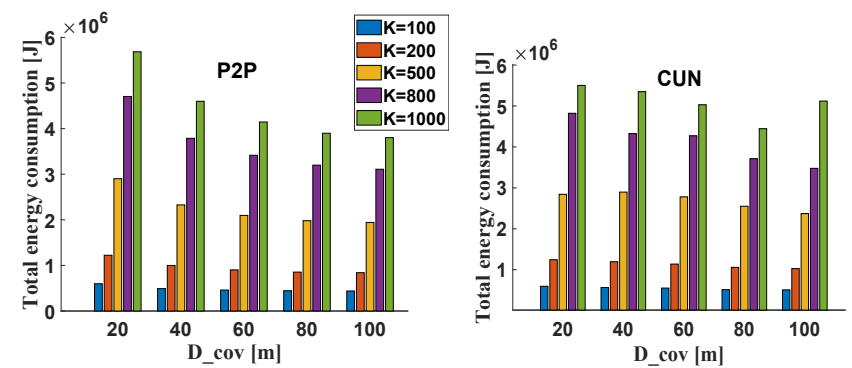

Fig. 4: The total energy consumption versus the coverage region in P2P and CUN schemes, at different density levels of sensor distribution.

4) The processing time: The processing time of $\mathrm{P} 2 \mathrm{P}$ and CUN approaches is illustrated as in Fig.5. As can be observed from this figure, the UAV-assisted system can save a lot of solving time by employing the CUN approach. For example, at $K=500$, CUN approach s more than 200 times faster than the P2P approach. The results also present that in the small number of IoT devices scenarios, the P2P approach experiences a stable execution time among different $D_{c o v}$ 
values while the processing time of the CUN method reduces as $D_{\text {cov }}$ increases.
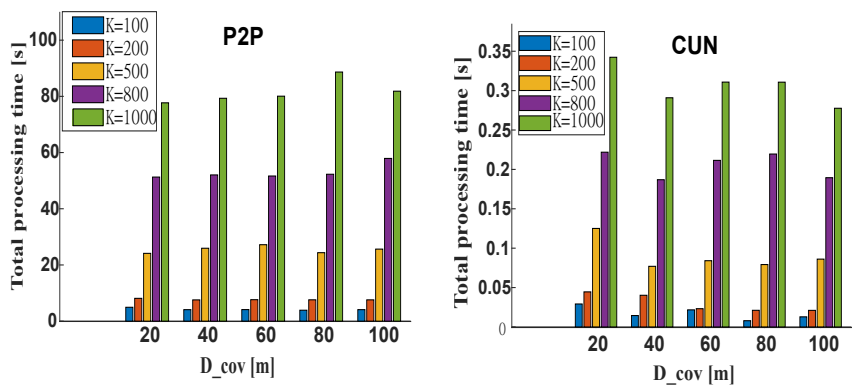

Fig. 5: The total processing time versus the coverage region in P2P and CUN schemes, at different density level of sensor distribution.

5) The number of UAVs: The QoS requirements of the system in terms of completing tracking the coverage region $\left(D_{\text {cov }}\right)$ and collecting data, are also investigated. To this end, we estimated the number of UAVs used in $\mathrm{P} 2 \mathrm{P}$ scheme and the number of clusters in CUN. When the number of IoT devices is as low as 50 or 250 , both schemes require the same minimum number of UAVs (1 and 4) to fulfil the mission. However, in large-scale scenarios such as 500, 750, and 1000 IoT devices, the number of UAVs used in the CUN approach is considerably smaller than that in the P2P method. Fig.6 depicts this comparison.

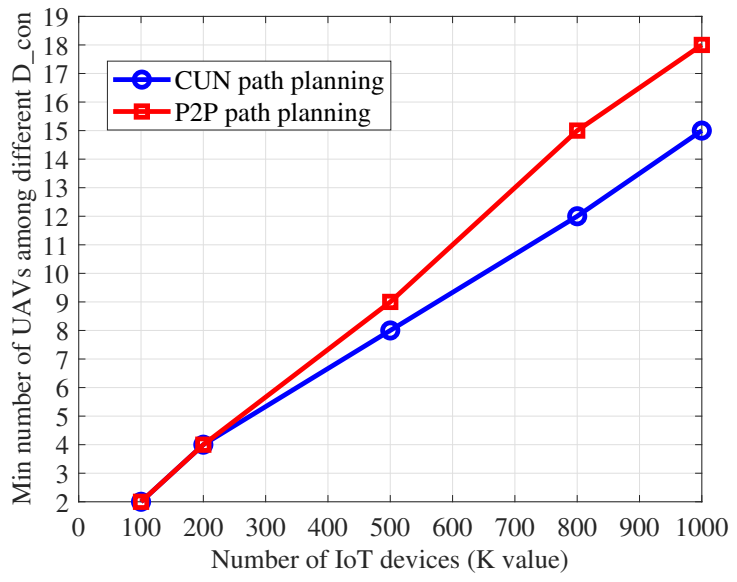

(a)

Fig. 6: The smallest number of UAVs used in the proposed methods at different $\mathrm{K}$ values.

In summary, P2P scheme outperforms CUN in terms of the total completion time, total energy consumption when the cover region increases or device density goes up. Conversely, CUN schemes have the advantage of faster processing time (i.e., in milliseconds) and use less UAVs. Hence, there is a trade-off between the two approaches with respect to time (processing time and completion time) and resources (energy consumption, UAV number) within a given set of requirements (QoS in terms of coverage area and device density level), which will merit further study for real-time applications, e.g., time-critical missions.

\section{CONCLUSIONS}

In this paper, we proposed to employ UAV-aided communications for intelligently tracking and gathering sensed data from IoT devices. We have proposed the practical embedded optimisation methods to support the time-critical applications. When the number of time slots, the number of UAVs, and the number of IoT devices are large, the non-convex optimisation problem become very complicated. We have demonstrated that our optimisation algorithms can be useful in large-scale scenarios. The proposed schemes with low-computational complexity to optimise the completion time and energy consumption can solve such complex optimisation problems in real-time.

\section{ACKNOWLEDGEMENTS}

This work was supported in part by the U.K. Royal Academy of Engineering (RAEng) under the RAEng Research Chair and Senior Research Fellowship scheme Grant RCSRF2021 $\backslash 11 \backslash 41$.

\section{REFERENCES}

[1] T. Q. Duong, L. D. Nguyen, and L. K. Nguyen, "Practical optimisation of path planning and completion time of data collection for UAVenabled disaster communications," in Proc. 15th Int. Wirel. Commun. Mob. Comput. Conf. (IWCMC 2019), Tangier, Morocco, 2019, pp. 372377.

[2] B. Li, Z. Fei, and Y. Zhang, "UAV communications for $5 \mathrm{G}$ and beyond: Recent advances and future trends," IEEE Internet Things J., vol. 6, no. 2, pp. 2241-2263, Apr. 2019.

[3] N. H. Motlagh, T. Taleb, and O. Arouk, "Low-altitude unmanned aerial vehicles-based internet of things services: Comprehensive survey and future perspectives," IEEE Internet Things J., vol. 3, no. 6, pp. 899922, Dec. 2016.

[4] C. Lin, G. Han, X. Qi, J. Du, T. Xu, and M. Martínez-García, "Energyoptimal data collection for unmanned aerial vehicle-aided industrial wireless sensor network-based agricultural monitoring system: A clustering compressed sampling approach," IEEE Trans. Ind. Informat., vol. 17, no. 6, pp. 4411 - 4420, 2021.

[5] H.-M. Chung, S. Maharjan, Y. Zhang, F. Eliassen, and K. Strunz, "Placement and routing optimization for automated inspection with UAVs: A study in offshore wind farm," IEEE Trans. Ind. Informat., vol. XX, no. X, pp. 1-1, 2020.

[6] D. A. Schoenwald, "AUVs: In space, air, water, and on the ground," IEEE Contr. Syst. Mag., vol. 20, no. 6, pp. 15-18, 2000.

[7] M.-N. Nguyen, L. D. Nguyen, T. Q. Duong, and H. D. Tuan, "Realtime optimal resource allocation for embedded UAV communication systems," IEEE Wireless Commun. Lett., vol. 8, no. 1, pp. 225-228, Feb. 2019.

[8] L. D. Nguyen, A. Kortun, and T. Q. Duong, "An introduction of real-time embedded optimisation programming for UAV systems under disaster communication," EAI Endorsed Transactions on Industrial Networks and Intelligent Systems, vol. 5, no. 17, pp. 1-8, Dec 2018.

[9] C. Zhan, Y. Zeng, and R. Zhang, "Energy-Efficient data collection in UAV enabled wireless sensor network," IEEE Wireless Commun. Lett., vol. 7, no. 3, pp. 328-331, Jun. 2018.

[10] G. Ahani, D. Yuan, and Y. Zhao, "Age-optimal UAV scheduling for data collection with battery recharging," IEEE Commun. Lett., vol. 25, no. 4, pp. 1254-1258, 2021.

[11] Z. Huang, C. Chen, and M. Pan, "Multiobjective UAV path planning for emergency information collection and transmission," IEEE Internet Things J., vol. 7, no. 8, pp. 6993-7009, 2020.

[12] M. B. Ghorbel, D. Rodriguez-Duarte, H. Ghazzai, M. J. Hossain, and H. Menouar, "Joint position and travel path optimization for energy efficient wireless data gathering using unmanned aerial vehicles," IEEE Trans. Veh. Technol., vol. 68, no. 3, pp. 2165-2175, 2019. 
[13] M. Samir, S. Sharafeddine, C. M. Assi, T. M. Nguyen, and A. Ghrayeb, "UAV trajectory planning for data collection from time-constrained IoT devices," IEEE Trans. Wireless Commun., vol. 19, no. 1, pp. 34-46, Jan. 2020.

[14] S. Wan, J. Lu, P. Fan, and K. B. Letaief, "Toward big data processing in IoT: Path planning and resource management of UAV base stations in mobile-edge computing system," IEEE Internet Things J., vol. 7, no. 7 , pp. 5995-6009, Jul. 2020.

[15] Y. Cai, Z. Wei, R. Li, D. W. K. Ng, and J. Yuan, "Joint trajectory and resource allocation design for energy-efficient secure UAV communication systems," IEEE Trans. Commun., vol. 68, no. 7, pp. 4536-4553, Jul. 2020.

[16] J. Hu, M. Jiang, Q. Zhang, Q. Li, and J. Qin, "Joint optimization of UAV position, time slot allocation, and computation task partition in multiuser aerial mobile-edge computing systems," IEEE Trans. Veh. Technol., vol. 68, no. 7, pp. 7231-7235, Jul. 2019.

[17] H.-S. Park and C.-H. Jun, "A simple and fast algorithm for K-medoids clustering," Expert systems with applications, vol. 36, no. 2, pp. 33363341, 2009.

[18] Y. Wu, K. H. Low, and C. Lv, "Cooperative path planning for heterogeneous unmanned vehicles in a search-and-track mission aiming at an underwater target," IEEE Trans. Veh. Technol., vol. 69, no. 6, pp. 6782 6787, Jun. 2020.

[19] M. Mozaffari, W. Saad, M. Bennis, and M. Debbah, "Mobile unmanned aerial vehicles (UAVs) for energy-efficient internet of things communications," IEEE Trans. Wireless Commun., vol. 16, no. 11, pp. 7574-7589, Nov. 2017.

[20] L. D. Nguyen, "Resource allocation for energy efficiency in 5G wireless networks," EAI Endorsed Transactions on Industrial Networks and Intelligent Systems, vol. 5, no. 14, pp. 1-7, Apr. 2018.

[21] Y. Zeng, X. Xu, and R. Zhang, "Trajectory design for completion time minimization in UAV-enabled multicasting," IEEE Trans. Wireless Commun., vol. 17, no. 4, pp. 2233-2246, Apr. 2018

[22] W. Khawaja, I. Guvenc, D. Matolak, U.-C. Fiebig, and N. Schneckenberger, "A survey of air-to-ground propagation channel modeling for unmanned aerial vehicles," IEEE Commun. Surveys Tuts., vol. 21, no. 3, pp. 2361 - 2391, May 2019.

[23] M. Alzenad, A. El-Keyi, and H. Yanikomeroglu, "3-D placement of an unmanned aerial vehicle base station for maximum coverage of users with different QoS requirements," IEEE Wireless Commun. Lett., vol. 7, no. 1, pp. 38-41, Feb. 2018.

[24] N. Lethaby, "Wireless connectivity for the internet of things: One size does not fit all," Texas Instruments, 2017.

[25] M. Grant and S. Boyd, "CVX: MATLAB software for disciplined convex programming, version 2.1," http://cvxr.com/cvx, Mar. 2014.

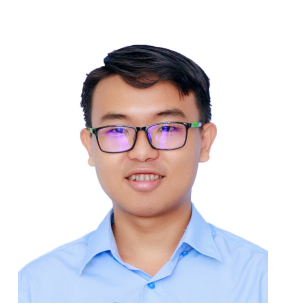

Dang Van Huynh (Graduate Student Member, IEEE) is currently a $\mathrm{PhD}$ student at the School of Electronics, Electrical Engineering and Computer Science (EEECS), Queen's University Belfast, UK. From Aug. 2017 to Dec. 2020, he worked as a Research Assistant at Faculty of Computer Networks and Communications, Vietnam National University HoChiMinh City, University of Information Technology (VNUHCM-UIT). He obtained the B.Eng. degree (honors) in Information Technology and the M.S. degree in Computer Science at VNUHCMUIT in 2017 and 2019, respectively. His main research interests include wireless communications, industrial Internet of Things, convex optimisation, and machine learning for wireless communications.

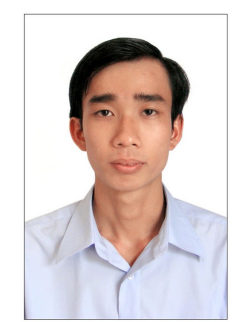

Tan Do-Duy received his B.S. degree from Ho Chi Minh City University of Technology (HCMUT), Vietnam, and M.S. degree from Kumoh National Institute of Technology, Korea, in 2010 and 2013, respectively. He received his $\mathrm{Ph} . \mathrm{D}$. degree from $\mathrm{Au}-$ tonomous University of Barcelona, Spain, in 2019 $\mathrm{He}$ is currently with the Department of Computer and Communication Engineering, Ho Chi Minh City University of Technology and Education (HCMUTE) in Vietnam as an Assistant Professor. His main research interests include wireless cooperative communications, real-time optimisation for resource allocation in wireless networks, and coding applications for wireless communications.

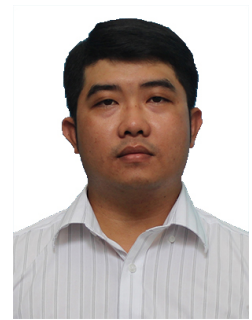

Long D. Nguyen (Member, IEEE) was born in Dong Nai, Vietnam. He received his Ph.D. degree in Electronics and Electrical Engineering from Queen's Univerisity Belfast (QUB), UK, in 2018. He was a Research Fellow at Queen's University Belfast, UK for a part of Newton project (2018-2019). He is currently with Dong Nai University in Vietnam as an Assistant Professor and Duy Tan University as an Adjunct Assistant Professor. His research interests include convex optimization techniques for resource management in wireless communications, energy efficiency approaches, and real-time embedded optimization for wireless networks and Internet of Things (IoTs). He was awarded the Best Paper Award at the IEEE Digital Signal Processing (DSP) 2017, the IEEE International Conference on Recent Advances in Signal Processing, Telecommunication and Computing (Sigtelcom) 2018, the IEEE International Conference on Communications (ICC) 2019, the International Wireless Communications \& Mobile Computing Conference (IWCMC) 2019 and the IEEE Global Communications Conference (GLOBECOM) 2019. He was also awarded the Exemplary Reviewer Award in IEEE Communications Letters 2018.

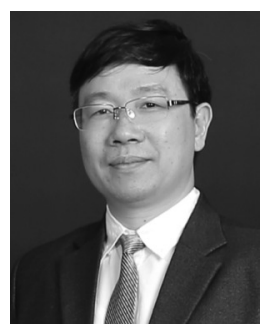

Minh-Tuan Le was born in Thanh Hóa, Vietnam, in 1976. He received the B.E. degree in electronic engineering from the Hanoi University of Science and Technology, Vietnam, in 1999, and the M.S. and $\mathrm{Ph} . \mathrm{D}$. degrees in electrical engineering from Information and Communication University (Department of Electrical and Engineering of Korean Advanced Institute of Science and Technology (KAIST), Daejon, South Korea), in 2003 and 2007, respectively. From 1999 to 2001 and from 2007 to 2008, he worked as a Lecturer with the Posts and Telecommunication Institute of Technology (PTIT), Vietnam. From November 2012 to 2015, he worked at the Hanoi Department of Science and Technology, Vietnam. He is currently working at the MobiFone Reasearch and Development Center, MobiFone Corporation, Vietnam. His research interests include space-time coding, space-time processing, and MIMO systems. He was a recipient of the 2012 ATC Best Paper Award. 


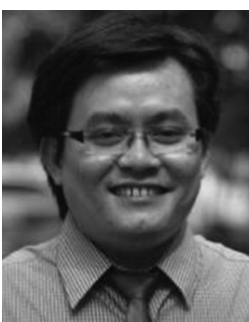

Nguyen-Son Vo received the Ph.D. degree in communication and information systems from the Huazhong University of Science and Technology, Wuhan, China, in 2012. He is currently with the Institute of Fundamental and Applied Sciences, Duy Tan University, Ho Chi Minh City, Vietnam. His research interests include self-powered multimedia wireless communications, quality of experience provision in wireless networks for smart cities, and Internet of Things for disaster and environment management. He was the recipient of the Best Paper Award at the IEEE Global Communications Conference 2016 and the prestigious Newton Prize 2017. He has been serving as an Associate Editor for the IEEE Communications Letters since 2019, Guest Editor for Elsevier Physical Communication in 2019, Guest Editor for IET Communications in 2018, and Guest Editor for ACM/Springer Mobile Networks and Applications in 2017 and 2018 .

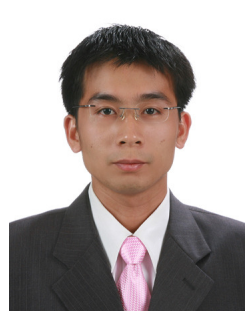

Trung Q. Duong (S'05, M'12, SM'13) is a Chair Professor in Telecommunications at Queen's University Belfast (UK). He also holds a prestigious Research Chair of Royal Academy of Engineering. His current research interests include wireless communications, machine learning, realtime optimisation, and data analytic.

Dr. Duong currently serves as an Editor for the IEEE TRANSACTIONS ON WIRELESS COMMUNICATIONS, IEEE TRANSACTIONS ON VEHICULAR TECHNOLOGY, and an Executive Editor for IEEE Communichtions Letters. He was awarded the Best Paper Award at the IEEE Vehicular Technology Conference (VTC-Spring) in 2013, IEEE International Conference on Communications (ICC) 2014, IEEE Global Communications Conference (GLOBECOM) 2016 and 2019, IEEE Digital Signal Processing Conference (DSP) 2017, and International Wireless Communications \& Mobile Computing Conference (IWCMC) 2019. He is the recipient of prestigious Royal Academy of Engineering Research Fellowship (2015-2020) and has won a prestigious Newton Prize 2017. 